\title{
Elmar Mittler zum 80. Geburtstag
}

https://doi.org/10.1515/bfp-2020-0021

\section{Spaziergang am Rhein}

Ein angenehmer sommerlicher Spaziergang mit Elmar Mittler am Ufer des Rheins vor mehr als vier Jahrzehnten war der Beginn unserer erfolgreichen, bis heute andauernden Kooperation und gewissermaßen die Geburtsstunde der BFP. Eine derartige Entwicklung haben wohl nicht viele Fachzeitschriften aufzuweisen. Sie ist vor allem dem jüngeren Kollegen zu verdanken, dessen vielfältige Initiativen seither im Inhalt der BPF dokumentiert wurden. Möge dieser Trend auch künftig anhalten!

Paul Kaegbein

\section{Menschlich wie fachlich bereichert}

Elmar Mittler lernte ich kennen, als er Sekretär der Arbeitsgruppe Bibliotheksplan Baden-Württemberg war. In dieser Funktion kam er in den 1970er-Jahren nach Stuttgart, um auch in der Bibliothek der Fachhochschule für Bibliothekswesen zu recherchieren. Das bot die Gelegenheit zu kollegialem Gedankenaustausch, der dazu führte, dass ich 1979 für den 3. Jahrgang von BIBLIOTHEK Forschung und Praxis einen ersten Beitrag lieferte. Als er mich einlud, ab 1985 mit dem 9. Jahrgang in das Herausgeberkollegium einzutreten, zögerte ich nicht lange, um mit der Übernahme dieser Aufgabe die freundschaftliche Zusammenarbeit weiter zu vertiefen. Sie hat mich in den vergangenen Jahrzehnten ebenso menschlich wie fachlich bereichert. Dafür bin ich überaus dankbar und wünsche Elmar, dass er noch lange die Bibliothekswelt mit seinem Wissen bereichert.

Peter Vodosek

\section{Vielfältige Aktivitäten und Pläne}

Lieber Elmar, Dein 80. Geburtstag fällt leider in die unbeliebten CoronaZeiten - das hatte ich Dir anders gewünscht! $\mathrm{Zu}$ diesem besonderen Geburtstag gratuliere ich Dir sehr herzlich und wünsche Dir neben bester Gesundheit als Grundlage wei- terhin so viel Freude und Energie an und für Deine so vielfältigen Aktivitäten und Pläne. Ich freue mich, dass sich BIBLIOTHEK - Forschung und Praxis so gut entwickelt hat.

\section{Herzlichste Grüße}

Monika Cremer

\section{Universalbibliothekar}

Es gibt, lieber Elmar, nicht nur Universalgelehrte, sondern auch Universalbibliothekare. Letzteres ist seltener als ersteres, so dass zu dieser Gruppe zu gehören eine große Ehre ist. In Deiner Karriere hast $\mathrm{Du}$, soweit ich weiß, kaum etwas ausgelassen, was Bibliothekare auszeichnet und vielfach ehrt. Von Anfang an hast Du die Weiterentwicklung von Bibliotheken geplant und das niemals aufgegeben. Für öffentliche und wissenschaftliche Bibliotheken hast Du stets das Gemeinsame gesehen und sie niemals auseinander dividiert. Du hast mehrfach modernisiert und Bibliotheksgebäude unumkehrbar wachgeküsst. Der bis dato größte Bibliotheksverbund in Deutschland geht auf Dich zurück und existiert noch immer. Die digitale Transformation historischer Bestände hast Du mit einem großen FuE-Department an der SUB nicht nur realisiert, sondern bis $\mathrm{zu}$ dem einzigen geisteswissenschaftlichen GRID- und dem nun einzigartigem Digital-Humanities-Projekt „TEXTGrid“ geführt. Mit dem Höhepunkt der „Palatina“ hast Du der Welt gezeigt, was sich mit Bibliotheken machen lässt, wenn man versteht, was in Bibliotheken steckt. Maßstäbe hast $\mathrm{Du}$ mit der Palatina und vielem Weiteren Erfolge gesetzt. Doch darf ich leider nicht das ganze Heft voll schreiben und schließe mit der Frage, was Du vielleicht doch ausgelassen haben könntest. Meine Antwort darauf ist, dass Dir als Universalbibliothekar das nicht passieren konnte: Wirklich großartig, lieber Elmar, und in der Tat bewundernswert!

Andreas Degkwitz

\section{Leitende Figur}

Elmar Mittler war Gründer und eine leitende Figur des German-North-American-Resources-Projekt (GNARP). Auch ich war fast von Anfang an dabei und einige Jahre nach 
der Gründung des Projektes hatten wir ein Treffen deutscher und US-amerikanischer Mitglieder in Göttingen. Die Amerikaner versuchten, deutsch $\mathrm{zu}$ reden und die Deutschen englisch. Weil das Resultat etwas chaotisch war, habe ich vorgeschlagen, dass jeder seine Muttersprache benutzt, weil alle beide Sprachen gut verstehen könnten. Elmar war mit Recht auf sein Englisch stolz und ist einige Zeit bei Englisch geblieben, bis die technischen Details so kompliziert geworden waren, dass auch er letztendlich zur Muttersprache gewechselt ist. Internationale Projekte sind oft schwierig und GNARP existiert immer noch.

Michael Seadle

\section{Ein Ver-MITTLER}

Seit 2005 bin ich im Herausgebergremium von BIBLIOTHEK - Forschung und Praxis. Das ist eine lange Zeit und es ist eine lange Zeit der Zusammenarbeit mit Elmar Mittler.

Wenn ich darüber nachdenke, wie man Elmar Mittler beschreiben könnte, was ihn auszeichnet und wie er seinerseits sein Umfeld prägt, dann fallen mir Stichworte ein wie Impulsgeber, Moderator, Netzwerkknüpfer, aber auch Antreiber und Streiter für seine Überzeugungen und vor allem für seine Zeitschrift. Ich habe ihn immer auch wahrgenommen als einen Wanderer zwischen unterschiedlichen Welten und er ist das, was schon in seinem Namen steckt: ein Ver-MITTLER! Herzlichen Glückwunsch, lieber Elmar!

\section{Cornelia Vonhof}

\section{Ein großer Schritt, der viele Erfolge gezeigt hat}

Es ist immer wieder eine große Freude, Elmar, Dir zu Deinen runden und unrunden Geburtstagen auf diese Weise $\mathrm{zu}$ gratulieren. Auch an Deinem 80. Geburtstag zeigt sich, dass Du weiterhin von der ,unheimlichen Aktivität' besessen bist, die mich schon bei Deiner Führung durch die „Palatina“-Ausstellung in Heidelberg 1986 fasziniert hat. Besondere Erinnerungen habe ich an Deine (und meine) Begeisterung für alle Schritte, die Bibliotheksverbände in Deutschland zu vereinen. Beim Göttinger Bibliothekskongress 1995 stimmten die Mitgliederversammlungen des VBB und des BBA für eine zukünftige
Vereinigung. Als ich kurz nach dieser Entscheidung vor den Hörsälen stand, stürztest du auf mich zu und fragtest, wie denn das Ergebnis der Abstimmung war. Als ich dir das positive Ergebnis mitteilte, umarmtest du mich spontan aus lauter Freude über diese Entscheidung. Dein begeistertes Engagement für einen einheitlichen Bibliotheksverband führte Dich zur Gründung der BDB, heute BID. Ein großer Schritt, der viele Erfolge gezeigt hat, auch wenn wir uns sicher gemeinsam wünschen, dass die noch vorhandenen bibliothekarischen Verbände sich noch einmal enger zusammenschließen könnten, um noch einheitlicher gegenüber der Politik aufzutreten. Danke Dir für jeden Schritt auf diesem Weg!

Claudia Lux

\section{... bringt die Community zusammen}

Lieber Elmar, wir beide wissen, warum ich Dir sehr viel lieber zum 79. gratulieren würde. Aber ich bin mir sicher: Auch nach Deinem 80. bleibst Du ein Zukunftsgestalter-Gestalter. Als Library-Avenger wärst Du Nick Fury - der altert nicht. Und er bringt die Community zusammen. Das werden wir auch in Zukunft brauchen. Deine Superkraft ist die Neugier, die hält jung. Glückwunsch zu inzwischen 80 Jahren Erfahrung mit dieser Welt. Und danke, dass Du diese Erfahrung so freigiebig mit uns teilst!

Deine Julia Bergmann

\section{Bibliotheks- und Informationswissenschaft neu aufgestellt}

Lieber Elmar, Als 2003 bis 2005 das Institut für Bibliotheks- und Informationswissenschaft der Humboldt-Universität zu Berlin, dessen Geschäftsführender Direktor ich damals war, wegen der einschneidenden Sparmaßnahmen an den Berliner Hochschulen von Schließung bedroht war, warst $\mathrm{Du}$ es, Elmar, der das Institut gerettet hat. Ich schlug dem Präsidium der Humboldt-Universität die Bildung einer externen Kommission unter Deiner Leitung vor, die das Institut evaluieren und eine Zukunftsperspektive entwickeln sollte. Du hast Dekan, Präsidium und Gremien überzeugt. Die Streichungspläne wurden gestrichen und das Institut nach Deinen Empfehlungen neu aufgestellt. 
Allein dies wäre ein exzellenter Grund zu feiern - Deinen 80. Geburtstag in Freude und Zuversicht zu begehen, gibt es unzählbar weitere Gründe.

Mit Dank und Gruß

Konrad Umlauf

\section{Elmar Mittler fast in Basel}

Lieber Elmar, Grüße aus der Universitätsbibliothek Basel. Aus der Stadt, zu der Du eine „Fast-Beziehung“ hast. Der Basler Altbundesrat Tschudi soll einmal ausgerufen haben: „Basel ist eine Fast-Stadt“. Was hat es damit auf sich? Nun ja, die Baslerinnen und Basler sind wahrlich immer „fast“ dabei, aber man steht sich nicht selten selbst im Weg zum Glück oder Erfolg.

- Schlacht von Sankt Jakob 1444: Die Basler Truppen warteten hinter der Mauer, man wollte die Entwicklung beobachten ... (Feierte aber den Pyrrhussieg mit!)

- Helvetik: Mit Peter Ochs hat die Stadt eine große intellektuelle Kapazität, die man dennoch ablehnt und „hinauswirft“.

- Den größten Flughafen der Schweiz: Stand zur Debatte diesen in Birsfelden zu errichten, wollten die Basler aber nicht.

- Sonderbundskrieg: Die Basler Truppen kamen erst, als die Schlacht vorbei war.

- Die berühmte Kunstsammlung Von Hirsch: Die Basler meinten, man solle sich keine Sammlung aufdrängen lassen (sie wurde dann Ende der 1970-er Jahre bei Sotherby's verkauft. Und die Basler kauften dann einige Exponate wieder zurück).

- Und eben, fast Elmar Mittler!

Ja, „Me hett nyt, me gitt nytt“ so das Motto des Basler Daigs.

Alles Gute zum Jubiläum wünscht Dir Alice Keller

\section{Open Access}

Open Access ist ein Thema, das Elmar Mittler früher als andere beschäftigt hat. So sagte er dem Deutschlandfunk bereits 2005 zur Literaturversorgung in der Medizin: „[W]enn der Staat oder irgendwelche sonstigen Fördereinrichtungen, wenn die Geld für wissenschaftliche Förderungen geben, dann muss dafür gesorgt sein [...], dass diese
Ergebnisse jedem Arzt, aber auch jedem Patienten im Zweifelsfall frei zugänglich sind“ [https://www.deutschlandfunk.de/informationen-fuer-alle.740.de.html?dram:a rticle_id=111397]. Bis heute kämpft er gewissenhaft und unermüdlich für einen besseren Zugang, zuletzt als Herausgeber der Zeitschrift BIBLIOTHEK -Forschung und Praxis. Dabei wird jeder Aspekt fein abgewogen. Das ursprünglich harmlose Konzept des Open Access ist inzwischen ein wahrhaftes transformatives Element im Publikationswesen, litt aber auch immer unter moralisierenden Debatten, deren Teilnehmende oft darin versagten, den jeweils betroffenen, konkreten Sinnzusammenhang der vielschichtigen Phänomene rund um Open Access herzustellen. Die Differenziertheit, mit der Elmar Mittler sich des Themas annimmt, ist vorbildlich.

Wolfram Horstmann

\section{Immer am Puls der Zeit}

Ich bewundere Elmar Mittler sehr, wie er tagesaktuelle Bibliotheksthemen stets präsent hat und vom Beginn seiner Tätigkeit für die Zeitschrift bis heute immer am Puls der Zeit ist.

Hannelore Vogt

\section{Ideensprühende und unermüdliche Institution}

Lieber Elmar, als ich 1994 mit dem Bibliothekswesen in Berührung kam, hattest $\mathrm{Du}$ mit der Badischen Landesbibliothek und der Universitätsbibliothek Heidelberg bereits zwei bedeutende Häuser geleitet und warst Direktor des dritten, der Niedersächsischen Staats- und Universitätsbibliothek Göttingen. Ein Vierteljahrhundert später bist Du in unserer Branche immer noch eine Institution, vielfach geehrt, ideensprühend und unermüdlich an der Arbeit. Ich freue mich, Dich als Mitherausgeber von BIBLIOTHEK -Forschung und Praxis regelmäßig zu treffen und zu sprechen und wünsche dir weiterhin solch waches Interesse für unseren wunderbaren Beruf, Gesundheit und Lebensfreude. Ad multos annos!

Achim Bonte 